\title{
VARIATIONAL INVARIANTS OF RIEMANNIAN MANIFOLDS
}

\author{
BY
}

JERROLD SIEGEL AND FRANK WILLIAMS

\begin{abstract}
This paper treats higher-dimensional analogues to the minimum geodesic distance in a compact Riemannian manifold $M$ with finite fundamental group. These invariants are based on the concept of homotopy distance in $M$. This defines a parametrized variational problem which is approached by globalizing the Morse theory of the spaces of paths between two points of $M$ to the space of all paths in $M$. We develop machinery that we apply to calculate the invariants for numerous examples. In particular, we shall observe that knowledge of the invariants for the standard spheres determines the question of the existence of elements of Hopf invariant one.
\end{abstract}

Introduction and background. One of the classical objects of study in Riemannian geometry is the notion of minimum geodesic distance in a manifold $M$. In this paper we shall conduct a systematic study of higher-dimensional analogues to this concept.

Just as the minimum geodesic distance is based on the lengths of paths in $M$, the invariants we shall consider are based on the widths of homotopies into $M$. (The width $|H|$ of a homotopy $H: X \times I \rightarrow M$ is the supremum of the lengths of the paths traversed by the individual points of $X$ under $H$.) As the geodesic distance dist $(x, y)$ between two points $x, y$ in the same component of $M$ is the infimum of the lengths of the paths connecting them, so may we consider, for homotopic maps $f, g$ : $X \rightarrow M$, the homotopy distance $w(f, g)$ which is the infimum of the widths of all homotopies from $f$ to $g$. We may think of this as a parametrized variational problem or as a minimal path problem in the function space $M^{X}$.

In general $w(f, g)$ is not finite. For example, let $e: \mathbf{R} \rightarrow S^{1}$ be the exponential map and $c: \mathbf{R} \rightarrow S^{1}$ be any constant map. It is easy to see that $w(e, c)=\infty$ since for any homotopy from $e$ to $c$ there are points that must "unwind" arbitrarily many times. The starting point of the body of work of which this paper forms part was the realization that this example represents the exceptional case. The more general situation is suggested by the following simple example.

0.1. EXAmple. (a) For any $f, g: \mathbf{R}^{2} \rightarrow S^{2}, w(f, g) \leqslant 2 \pi$.

(b) There exist maps $f^{\prime}, g^{\prime}: \mathbf{R}^{2} \rightarrow S^{2}$ such that $w\left(f^{\prime}, g^{\prime}\right)=2 \pi$.

Proof. (a) Let $h: S^{3} \rightarrow S^{2}$ be the Hopf map which has dilatation 2 [11]. Lift $f$ and $g$ to $\bar{f}, \bar{g}: \mathbf{R}^{2} \rightarrow S^{3}$. For dimensional reasons, we may deform $\bar{f}$ to $\bar{g}$ by homotopies of

Received by the editors March 24, 1983 and, in revised form, October 3, 1983.

1980 Mathematics Subject Classification. Primary 53C20, 55R65; Secondary 58E05, 55P99.

Key words and phrases. Riemannian manifold, homotopy, Morse theory, fibrations. 
widths arbitrarily close to $\pi$. We thus obtain, by composition with $h$, a homotopy from $f$ to $g$ of width arbitrarily close to $2 \pi$.

(b) By wrapping $\mathbf{R}^{2}$ around $S^{2}$ along rays from the origin, we obtain a map $f_{1}$. Elementary algebraic topology yields that any homotopy from $f_{1}$ to the constant map $g_{1}$ at the origin must move some point along a path of length at least $2 \pi$.

This example indicates that using the homotopy distance between maps into $M$ as the analogue of the minimum geodesic distance between points of $M$ produces meaningful information for certain domain spaces $X$. This phenomenon turns out to be quite general. In fact, the best description of the general situation depends only on the dimension of the domain space $X$. For the rest of the paper, $M$ will denote $a$ compact Riemannian manifold with $\pi_{1}(M)$ finite. By the dimension of a normal space we mean its covering dimension. The fundamental existence theorem is

0.2. THEOREM [2]. For each integer $k \geqslant 0$ there exists a real number $\tilde{b}_{k}(M)$ such that if $X$ is any normal space of dimension $\leqslant k$ and $f \sim g: X \rightarrow M$, then $w(f, g) \leqslant$ $\tilde{b}_{k}(M)$. Hence there are defined a sequence of invariants $b_{k}(M)=$ infimum of such $\tilde{b}_{k}(M)$.

It was also shown in [2] that these numbers are nontrivial: in fact, $\lim _{k \rightarrow \infty}\left(b_{k}(M)\right)=\infty$. The numbers reflect subtle topological and geometrical properties of $M$. A good example of this is the fact that $b_{2 n-2}\left(S^{n}\right)=2 \pi$ if and only if $n=2,4$ or 8 (otherwise $b_{2 n-2}\left(S^{n}\right)=3 \pi$ ). As one might suppose, the difference in the calculations of the two cases involves the existence of elements of Hopf invariant one.

Our present study involves two separate techniques, one for producing upper bounds and one for lower bounds. We shall observe that the method for studying lower bounds applies to all manifolds. Our technique for estimating upper bounds presents a different situation. The existence of the numbers $b_{k}(M)$ was demonstrated by identifying a compact subset $C_{k}$ of paths of finite length in the full space of paths $M^{I}$. This subspace possessed sufficient lifting properties to assure us of the fact that any homotopy, regarded as a map $X \rightarrow M^{I}$, could be deformed into $C_{k}$ keeping the end maps fixed. On the other hand, our explicit computations in [ 3 and 10] depended on finding subspaces of $M^{I}$ with much stronger lifting properties. We are now able to give a fairly complete answer as to what class of manifolds possesses path spaces with these stronger properties. A sufficient condition is that $M$ has everywhere positive Ricci curvature. We shall also produce a manifold (with negative curvature) whose pathspace does not possess subsets with the stronger lifting properties.

We shall review this material In $§ I$, which contains precise definitions and statements of results as well as a number of examples, including a proof independent of a priori knowledge of the Hopf invariant one dimensions that $b_{2 n-2}\left(S^{n}\right)=2 \pi$ implies the existence of an element of Hopf invariant one in $\pi_{2 n-1}\left(S^{n}\right)$.

Applications now also include complete calculations of the invariants for standard spheres, products of spheres with different radii, and the real, complex and quaternionic projective spaces. It is also possible to compute all but a finite number of the invariants for comnact $I$ ie ornuns $W_{e}$ shall present these calculations in $\S I I$. 
I. Definitions and statement of results. The results in this paper are best stated in terms of three sequences of extended real numbers. We begin this section by defining these sequences. For convenience we shall assume that our domain spaces $X$ are finite-dimensional complexes. By the "bridge" technique of Morita [9] our results apply without change to all finite-dimensional normal spaces. We recall the following definitions from [10]. Since they can be stated for any metric space, we temporarily drop our standing hypotheses on $M$.

1.1 Definitions. Let $(M, d)$ be a metric space.

(a) The length $|\sigma|$ of a paht $\sigma: I \rightarrow M$ is given by the formula

$$
|\boldsymbol{\sigma}|=\sup \left\{\sum_{i=1}^{k} d\left(\sigma\left(t_{i}\right), \boldsymbol{\sigma}\left(t_{i-1}\right)\right)\right\}
$$

taken over all partitions $0=t_{0}<t_{1}<\cdots<t_{k}=1$, for all $k$.

(b) The width $|H|$ of a homotopy $H: X \times I \rightarrow M$ is given by the formula $|H|=\sup \left\{\left|H_{x}\right|: x \in X\right\}$.

For maps $f, g: X \rightarrow M$ and a homotopy $H$ from $f$ to $g$, we set:

(c) $W(H)=\inf \left\{\left|H^{\prime}\right|: H^{\prime} \sim H\right.$ rel $\left.X \times\{0,1\}\right\}$.

(d) $w(f, g)=\inf \left\{\left|H^{\prime}\right|: H_{0}^{\prime}=f, H_{1}^{\prime}=g\right\}$.

Finally we arrive at the two basic sequences:

(e) $B_{q}(M)=\sup \{W(H): H: X \times I \rightarrow M, \operatorname{dim}(X) \leqslant q\}$.

(f) $b_{q}(M)=\sup \{w(f, g): f \sim g: X \rightarrow M, \operatorname{dim}(X) \leqslant q\}$.

Thus if $\operatorname{dim}(X) \leqslant k$ then any homotopy $H: X \times I \rightarrow M$ can be deformed relative to the end maps to one of width $<B_{k}(M)+\varepsilon$ for any $\varepsilon>0$. Also, for any homotopic maps $f, g: X \rightarrow M$, there exists a homotopy between $f$ and $g$ of width $<b_{k}(M)+\varepsilon$. Of course $B_{k}(M)$ and $b_{k}(M)$ are, in general, only extended real numbers, but if $M$ is a compact Riemannian manifold with $\pi_{1}(M)$ finite it was shown in [2 and 10] that these numbers are finite. In describing our results it will be useful to relate these sequences to a third one, a relative version of $B_{k}(M)$, that appears to be finite only in much more restricted settings.

1.2. Definitions. (a)A number $R>0$ is called a relative $k$-bound for $M$ if for any $X, \operatorname{dim}(X) \leqslant k$, any homotopy $H: X \times I \rightarrow M$, and any subcomplex; $A \subset X$ for which $|H|_{A} \mid<R$, there exists a deformation $H^{\prime} \sim H \operatorname{rel}(X \times\{0,1\})$ with $\left|H^{\prime}\right|<R$ and for each stage $H^{s}$ of the deformation we have $\left|H^{s}\right|_{A} \mid<R$.

(b) Define $B_{k}^{\#}(M)=\inf \{R: R$ is a relative $k$-bound for $M\}$.

Obviously $b_{k}(M) \leqslant B_{k}(M) \leqslant B_{k}^{\#}(M)$. As we stated above $B_{k}(M)$ is finite whenever $M$ satisfies our standing hypotheses. The situation is more complicated for the $B_{k}^{\#}(M)$. We will present examples of spaces (spheres with appropriate geometries) for which $B_{k}^{\#}(M)=\infty$. On these manifolds there are thus homotopies that may be shortened to a certain width but not without first lengthening them on an already short part.

We begin to develop the technical machinery.

1.3. Definitions. (a) Give a fibration $F \subset E \rightarrow B$, a subset $C \subset E$ is called a $k$-carrier for the fibration if for every $X$ of dimension $\leqslant k$ and every pair of maps $f$ : $X \rightarrow B$ and $g: X \rightarrow E$ such that $p g=f$ we may deform $g$ vertically to a map $g^{\prime}$ such that $g^{\prime}(X) \subset C$. 
(b) $C$ is called a relative $k$-carrier if for every subcomplex $A \subset X$ and every map of pairs $g:(X, A) \rightarrow(E, C)$ such that $p g=f, g$ may be deformed vertically as a map of pairs to a map $g^{\prime}$ such that $g^{\prime}(X, A) \subset(C, C)$.

1.4. Theorem [2]. Let $F \subset E \rightarrow B$ be a Hurewicz fibration, locally trivial in the sense of Dold [6]. Let $B$ be compact and $F$ have the homotopy type of a CW-complex of finite type. Then for each $k \geqslant 0, E$ has a compact $k$-carrier.

1.5. COROLlaRY. Under the standing hypotheses on $M$, i.e. that $M$ is a compact Riemannian with finite fundamental group, $B_{k}(M)<\infty$.

Proof. We give a different proof of this corollary from the one given in [2] since we shall wish to refer to this modification in the sequel. Let $k \geqslant 0$. We begin by applying 1.4 to the fibration $\Omega M \subset M^{I} \stackrel{p}{\rightarrow} M \times M$, where $p(f)=(f(0), f(1))$, to find a compact $k$-carrier $C \subset M^{I}$. We form a new space $E$ by metrizing the space of piecewise smooth paths in $M$ as in $\$ 17$ of [8] so that the path-length function becomes continuous. The inclusion $i: E \rightarrow M^{I}$ is a homotopy equivalence over $M$ and the specific inverse $h$ defined in [8] does not increase path length. The compact set $h(C)$ is contained in the subset $E_{\lambda} \subset E$ consisting of paths of length $<\lambda$, for some $\lambda$. Hence $i(h(C)) \subset\left(M^{I}\right)_{\lambda}$. Since ih is vertically homotopic to the identity map of $M^{I}$, it follows that $i(h(C))$ is again a $k$-carrier.

Since any superset of a $k$-carrier is clearly a $k$-carrier, this proof of 1.5 shows that for every $k \geqslant 0$, there exists $\lambda$ such that $\left(M^{I}\right)_{\lambda}$ is itself a $k$-carrier.

We next consider the corresponding situation for $B_{k}^{\#}(M)$. The important difference is that the finiteness of $B_{k}^{\#}(M)$ imposes strong topological conditions on $M^{I}$. The following theorem summarizes the situation.

1.6. Theorem. Let $B_{k}^{\#}(M)<\lambda$. Then there exists $\lambda^{\prime} \leqslant \lambda$ such that $\left(M^{\prime}\right)_{\lambda^{\prime}}$ is a relative $k$-carrier. For such a $\lambda^{\prime}, \pi_{i}\left(\Omega(x, y), \Omega_{\lambda^{\prime}}(x, y)\right)=0$ for $i \leqslant k$ and all $(x, y) \in$ $M$.

Proof. The first implication is just a restatement of the definitions. The second results from applying the definition to representatives of the generators of the relative homotopy groups.

The general philosophy that we shall use in our computations goes as follows. Since any homotopy may be approximated by one that moves points along piecewise smooth paths, we shall henceforth use the notation $M^{I}$ to denote this subspace of the full pathspace. For $x, x^{\prime} \in M$ and $\lambda \geqslant 0$ the Morse theory [8] gives topological information about the space $\Omega_{\lambda}\left(x, x^{\prime}\right)$ of piecewise smooth paths in $M$ from $x$ to $x^{\prime}$ of length less than $\lambda$. A map $X \rightarrow \Omega_{\lambda}\left(x, x^{\prime}\right)$ may be considered to be a homotopy of width less than (or equal to) $\lambda$ between the constant maps of $X$ to $x$ and $x^{\prime}$, respectively. (This point of view is taken by Gromov in [7].) A reasonable approach to take in studying homotopies of width less than $\lambda$ between arbitrary maps is to globalize the Morse Theory for $\Omega_{\lambda}\left(x, x^{\prime}\right)$ to the map $p_{\lambda}:\left(M^{I}\right)_{\lambda} \rightarrow M \times M$, where $p_{\lambda}$ is the projection of the space of all piecewise smooth paths of length less than $\lambda$ to their endpoints. 
The calculations in [10] of the $B_{k}\left(S^{n}\right)$ were actually calculations of the $B_{k}^{\#}\left(S^{n}\right)$. That the situation becomes more complicated for other manifolds is seen through the following example.

1.7. Example (The Hourglass). We produce a $M$ such that $B_{1}^{\#}(M)=\infty$. Let $K$ be the surface in $\mathbf{R}^{3}$ given in cylindrical coordinates by the equations $r=z^{2}+1$, $|z| \leqslant 2$. Form $M$ by smoothly attaching 2-cells to each end of $K$ and extend the induced Riemannian metric on $K$ to $M$ in any way. Clearly

$$
B_{1}^{\#}(M) \geqslant B_{0}^{\#}(M) \geqslant \operatorname{diam}(M)=\max _{x, y \in M} d(x, y)>2 \pi .
$$

Let $\lambda>2 \pi$ and let $x=(1,0,0)$. We shall show that $\Omega_{\lambda}(x, x)$ is not connected and hence $\pi_{1}\left(\Omega(x, x), \Omega_{\lambda}(x, x)\right) \neq 0$. Choose $n$ so that $2(n+1) \pi \geqslant \lambda>2 n \pi$. Let $\sigma \in$ $\Omega_{\lambda}(x, x)$ wrap around the circle $z=0 n$ times. We observe that $\sigma$ is not homotopic in $\Omega_{\lambda}(x, x)$ to the constant path, since there is not enough "room" to pull it over the end. It follows, using Theorem 1.6, that $B_{1}^{\#}(M)>\lambda$. Since $\lambda$ was arbitrary, $B_{1}^{\#}(M)=\infty$.

1.8. EXAMPLE. It is instructive to try adjusting 1.7 by letting $M$ be the flat cylinder $r=1,|z| \leqslant L$, with its ends capped off. Again let $x=(1,0,0)$. In this case, for any $\varepsilon>0$ there exists $n$ such that the loop $\sigma_{n}(t)=(\cos (2 \pi n t), \sin (2 \pi n t), 0)$ is contractible in $\Omega_{2 \pi n+\varepsilon}(x, x)$. To see this, choose $m$ such that $m \sqrt{4 \pi^{2}+(L / m)^{2}}<2 \pi m+\varepsilon$ and set $n=2 m+1$. Consider the following diagram:

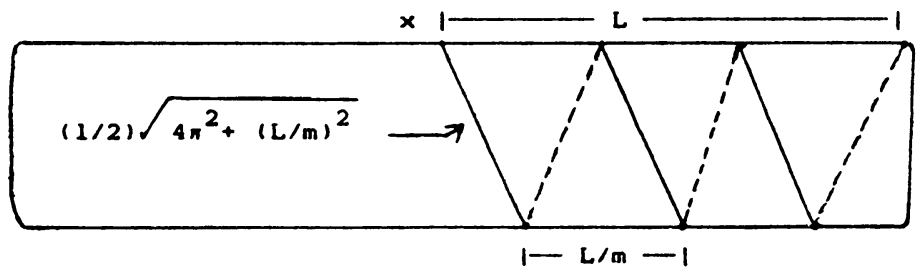

By pulling the $n$ loops toward the end we can get out and back in $2 m$ loops with one loop left over to slip over the end.

We do not known whether Example 1.8 possesses a compact relative $k$-carrier. The general area of questions dealing with the relationship of curvature and the existence of relative $k$-carriers seems to be a fruitful one for future work.

Because of Example 1.7 one might be tempted to dismiss the numbers $B_{k}^{\#}(M)$ from further consideration. In fact, in all the cases for which we have been able to compute the numbers $B_{k}$ and $b_{k}$, not only are the $B_{k}^{\#}$ finite but they are equal to the $B_{k}$. We shall be particularly concerned with when and why the various inequalities among these invariants become equalities. Our attention was drawn to this question by the surprising situation for the standard spheres. Here, for $n \geqslant 2$, we have the equality $B_{k}\left(S^{n}\right)=B_{k}^{\#}\left(S^{n}\right)$. Moreover, $b_{k}\left(S^{n}\right)=B_{k}\left(S^{n}\right)$ except when the Hurewicz map $\pi_{k}\left(\Omega S^{n}\right) \rightarrow H_{k}\left(S^{n}\right)$ is a nonzero epimorphism (i.e. when $k=n-1$ or $n=2,4$ or 8 and $k=2 n-2)$. In these cases $b_{k}\left(S^{n}\right)=B_{k-1}\left(S^{n}\right)$. We conjectured in [10] 
that the value of $b_{k}(M)$ is always either $B_{k}(M)$ or $B_{k-1}(M)$. Since the $B_{k}$ have been considerably easier to compute and describe than the $b_{k}$, the affirmative proof of this conjecture would represent a considerable help in the computation of the $b_{k}$. We have not yet been able to verify this conjecture, although all the computations we are now able to make are consistent with it. To lay the groundwork for these computations, we now begin listing the ingredients of the main results of this paper.

1.9. Let $x, x^{\prime} \in M, \lambda \geqslant 0, k$ be a nonnegative integer, and let

$$
\begin{aligned}
& \text { (a) } i_{*}: H_{k}\left(\Omega_{\lambda}\left(x, x^{\prime}\right)\right) \rightarrow H_{k}\left(\Omega\left(x, x^{\prime}\right)\right), \\
& \text { (b) } i_{\#}: \pi_{k}\left(\Omega_{\lambda}\left(x, x^{\prime}\right)\right) \rightarrow \pi_{k}\left(\Omega\left(x, x^{\prime}\right)\right)
\end{aligned}
$$

be induced by inclusion.

Let $\rho: H_{k}\left(\Omega\left(x, x^{\prime}\right)\right) \rightarrow H_{k}\left(\Omega\left(x, x^{\prime}\right)\right) /($ spherical elements) be projection.

In $§ I V$ we shall prove

1.10. THEOREM. Let $x, x^{\prime} \in M$.

(a) If $i_{*}$ fails to be onto, then $B_{k}(M) \geqslant \lambda$ and $b_{k+1}(M) \geqslant \lambda$.

(b) If $\rho i_{*}$ fails to be onto, then $b_{k}(M) \geqslant \lambda$.

(c) If $i_{*}$ fails to be a split epimorphism, then $b_{k+1}(M) \geqslant \lambda$.

Although homology conditions are usually easier to check, Theorem 1.10 also has a homotopy version:

1.11. THEOREM. Let $x, x^{\prime} \in M$.

(a) If $i_{\#}$ is not onto, then $B_{k}(M) \geqslant \lambda$ and $b_{k+1}(M) \geqslant \lambda$.

(b) If $i_{\#}$ fails to be a split epimorphism, then $b_{k+1}(M) \geqslant \lambda$.

The upper bounds obtained for $B_{k}\left(S^{n}\right)$ were based on the approximate covering homotopy property. We recall that a map $p: E \rightarrow B$ has the $\mathrm{XCHP}(k)$ provided that for any (normal) space $X$ of dimension $k$ or less, maps $H: X \times I \rightarrow B$ and $g: X \rightarrow E$ such that $p(g(x))=H(x, 0)$, and $\varepsilon>0$, there exists $G: X \times I \rightarrow E$ such that $G(x, 0)=g(x)$ and $p(G(x, t))$ is within $\varepsilon$ of $H(x, t)$. The connection between $B_{k}^{\#}(M)$ and the $\mathrm{XCHP}(k)$ is given by

1.12. THEOREM (CF. THEOREM 2.1 OF [10]). If $\left(M^{I}\right)_{\lambda} \rightarrow M \times M$ has the XCHP $(k)$, then $B_{k}^{\#}(M) \leqslant \lambda$. Hence $B_{k}(M) \leqslant \lambda$.

The necessary observation required to get the result for $B^{\#}$ rather than $B$ is that the $\mathrm{XCHP}(k)$ implies a relative $\mathrm{XCHP}(k)$ for pairs of complexes. The same argument that is used to show that this is true for fibrations applies also in this case.

1.13. REMARK. It is easy to show by tracking through the definitions that if $B_{k}^{\#}(M) \leqslant \lambda$ then $\left(M^{I}\right)_{\lambda} \rightarrow M \times M$ has the $\mathrm{XCHP}(k-1)$. Since we shall make no use of this fact, we omit its proof.

There are various sets of sufficient conditions for a map to have the $\operatorname{XCHP}(k)$ (see, e.g., [5 and 4]). For our purposes we shall prove the following theorem which makes use of the homotopy of the $\Omega_{\lambda}\left(x, x^{\prime}\right)$. 
1.14. THEOREM. Let $k \geqslant 1$ and $\lambda>0$. If there exists a dense set of points $\left\{\left(x, x^{\prime}\right)\right\} \subset M \times M$ such that

$$
\pi_{i}\left(\Omega_{\lambda^{\prime}}\left(x, x^{\prime}\right)\right) \rightarrow \pi_{i} k\left(\Omega\left(x, x^{\prime}\right)\right)
$$

is isomorphic for all $0 \leqslant i<k, \lambda^{\prime}<\lambda$, then $\left(M^{I}\right)_{\lambda} \rightarrow M \times M$ has the $\mathrm{XCHP}(k-1)$. Consequently $B_{k}^{\#}(M) \leqslant \lambda$.

A reasonable additional hypothesis is that the morphism be epimorphic on $\pi_{k}$, but this is not sufficient to guarantee the $\mathrm{XCHP}(k)$. However, this requirement is sufficient for $B_{k}^{\#}(M)$ :

1.15. THEOREM. If we add to the hypotheses of 1.14 the requirement that the maps $\pi_{k}\left(\Omega_{\lambda^{\prime}}\left(x, x^{\prime}\right)\right) \rightarrow \pi_{k}\left(\Omega\left(x, x^{\prime}\right)\right)$ be epimorphisms, then $B_{k}^{\#}(M) \leqslant \lambda$.

Under the hypotheses of Theorem 1.14 we have a completely determined situation for $B_{k}(M)$ and $B_{k}^{\#}(M)$ :

1.16. Corollary. Suppose $k$ and $\lambda$ satisfy the hypotheses of Theorem 1.14. If $\pi_{k}\left(\Omega_{\lambda^{\prime}}\left(x, x^{\prime}\right)\right) \rightarrow \pi_{k}\left(\Omega\left(x, x^{\prime}\right)\right)$ fails to be onto for any $x, x^{\prime} \in M$ and any $\lambda^{\prime}>\lambda$, then $B_{k}(M)>\lambda$. Otherwise $B_{k}^{\#}(M) \leqslant \lambda$.

Since $B_{k}(M) \geqslant b_{k}(M)$, upper bounds for the $B_{k}(M)$ are necessarily upper bounds for the $b_{k}(M)$. An additional upper bound for $b_{k}(M)$ appears in the following result.

1.17. TheOREM. Let $B_{k-1}(M) \leqslant \lambda$ and let $i_{*}: \pi_{k-1}\left(\Omega_{\lambda^{\prime}}\left(x, x^{\prime}\right)\right) \rightarrow \pi_{k-1}\left(\Omega\left(x, x^{\prime}\right)\right)$ be isomorphic for a dense set $\Sigma$ of $\left(x, x^{\prime}\right)$ 's and for all $\lambda^{\prime}>\lambda$. Then $b_{k}(M) \leqslant \lambda$.

1.18. REMARK. The nonexistence of elements of Hopf invariant one was used to show that $b_{2 n-2}\left(S^{n}\right) \geqslant 3 \pi$ for $n \neq 2,4$ or 8 . Theorem 1.17 provides the reverse implication. That is, if $b_{2 n-2}\left(S^{n}\right)>2 \pi$, then the suspension $\Sigma: \pi_{2 n-3}\left(S^{n-1}\right) \rightarrow$ $\pi_{2 n-2}\left(S^{n}\right)$ fails to be injective, since $\Omega_{2 \pi}\left(x, x^{\prime}\right) \sim S^{n-1}$ and the inclusion $S^{n-1} \subset \Omega S^{n}$ induces $\Sigma$. Hence there is no element of Hopf invariant one in $\pi_{2 n-1}\left(S^{n}\right)$. Thus if an alternate proof that $b_{2 n-2}\left(S^{n}\right)>2 \pi(n \neq 2,4$ or 8$)$ could be found, perhaps of a geometric nature, it would then provide an alternate proof of the nonexistence of elements of Hopf invariant one.

The setting of Theorem 1.15 provides us with one final lower bound theorem for $b_{k}(M)$ :

1.19. Corollary (TO TheOREM 1.10(b)). Under the hypotheses of Theorem 1.15 suppose that $\{0\} \neq \operatorname{ker}(h) \cap \operatorname{ker}\left(i_{\#}\right)$ in dimension $k-1$ for some one of the $\left(x, x^{\prime}\right)^{\prime}$ s. Then $b_{k}(M) \geqslant \lambda$.

To organize these results, it is useful to suppose that $\lambda$ is such that $\pi_{j}\left(\Omega, \Omega_{\lambda^{\prime}}\right)=0$ for all $\lambda^{\prime}>\lambda$.

1.20. Summary. Suppose that $\pi_{j}\left(\Omega\left(x, x^{\prime}\right), \Omega_{\lambda^{\prime}}\left(x, x^{\prime}\right)\right)=0$ (or, equivalently (in the 1-connected case $)$ that $\left.H_{j}\left(\Omega\left(x, x^{\prime}\right), \Omega_{\lambda^{\prime}}\left(x, x^{\prime}\right)\right)=0\right)$ for a dense set of $\left(x, x^{\prime}\right)$, for all $\lambda^{\prime}>\lambda$, and $j \leqslant k-1$. Then $B_{k-1}(M) \leqslant \lambda$. Furthermore: 
(i) if $i_{*}$ is an isomorphism on $\pi_{k-1}$, then $b_{k}(M) \leqslant \lambda$;

(ii) if $i_{*}$ does not split or if $\operatorname{ker}\left(i_{\#}\right) \cap \operatorname{ker}(h) \neq 0$ in dimension $k-1$ for any $\left(x, x^{\prime}\right)$ and any $\lambda^{\prime}>\lambda$, then $b_{k}(M)>\lambda$; and

(iii) if $i_{*}$ is not onto in dimension $k$ for some $\left(x, x^{\prime}\right)$ and any $\lambda^{\prime}>\lambda$, then $B_{k}(M)>\lambda$.

In general, $\pi_{j}\left(\Omega\left(x, x^{\prime}\right), \Omega_{\lambda^{\prime}}\left(x, x^{\prime}\right)\right)$ may never be trivial. Indeed, $\left(M^{I}\right)_{\lambda} \rightarrow M \times M$ may never have the $\mathrm{XCHP}(k)$ for any $\lambda$ or $k$. Remark 1.13 shows that 1.7 provides an example of such a $M$. This defines the limit of the information on upper bounds that is obtainable from the Morse theory. There are, therefore, Morse-theoretical lower bounds that always apply and upper bounds that always are finite but do not necessarily come from Morse theory. At this stage, information of a geometrical nature is necessary to go further. If the Ricci curvature $K(U, U)$ is positive for all unit tangent vectors on $M$, then for every $k$ there exists a $\lambda$ such that the hypotheses of 1.20 are satisfied [8]. In this case the situation is completely determined except when, in dimension $k-1, i_{*}$ does split and $\operatorname{ker}\left(i_{*}\right) \neq 0$ while $\operatorname{ker}\left(i_{\#}\right) \cap \operatorname{ker}(h)=0$. We shall see in the next section that in many instances this situation does not arise. Thus for these examples we obtain a complete computation of the $b_{k}(M)$ 's.

II. Examples and computations. We shall begin this section with three examples that illustrate various aspects of Theorems 1.10 and 1.15. In the first two we shall take $M$ to be a product of standard spheres, perhaps with different radii. We endow $M$ with the product metric. Thus the energy function on the product is the sum of the energy functions of the factors. Hence the critical paths are products of the geodesics on the factors and the index of a critical path is just the sum of the indices of its factors. Finally, the cells of $\Omega M$ correspond to the expected products of cells of the factors under the Morse-theoretic cell decomposition.

2.1. EXAMPLE. Let $M=S^{m} \times S^{n}, m<n$, both spheres with radius one. Using the above remarks, one calculates that the range of values where the morphism $\pi_{i}\left(\Omega_{\lambda}\left(x, x^{\prime}\right)\right) \rightarrow \pi_{i}\left(\Omega\left(x, x^{\prime}\right)\right)$ is an isomorphism is determined by only the cells of $\Omega\left(S^{m}\right)$. These are the cells that occur in the lowest dimensions relative to $\lambda$. The pattern of equalities and inequalities for $b_{k}(M), B_{k}(M)$ and $B_{k}^{\#}(M)$ are thus also those of $S^{m}$. The numerical values are, if $m \neq 2,4$ and 8 ,

$$
\begin{gathered}
B_{k}(M)=B_{k}^{\#}(M)=\sqrt{q^{2}+1} \pi, \quad(q-1)(m-1) \leqslant k<q(m-1), \\
b_{k}(M)=B_{k}(M), \quad k \neq m-1, \\
b_{m-1}(M)=\sqrt{2} \pi=B_{m-2}(M)=<B_{m-1}(M)=\sqrt{5} \pi .
\end{gathered}
$$

If we choose $m$ and $n$ such that $m-1 \neq 0(\bmod n-1)$, then $H_{n-1}(\Omega M)$ is spherically generated so that $\rho i_{*}$ is automatically onto when $i_{*}$ is, yet $b_{n-1}(M)=$ $B_{n-1}(M)$. Thus the converse of $1.10(\mathrm{~b})$ does not hold.

2.2. EXAmple. Let $M=S^{m} \times S^{n}, m<n<2 m-1$, but now let $S^{n}$ have radius 2 while $S^{m}$ retains radius one. The second factor now dominates, but with some 
influence from the first. The values now are, for $m, n \neq 2,4$ or 8 ,

$$
\begin{gathered}
B_{k}^{\#}(M)=B_{k}(M)=\left(\begin{array}{lc}
2 \pi \sqrt{q^{2}+1}, & 7 q-3 \leqslant k<7 q, \\
\pi \sqrt{4 q^{2}+8 q+5}, & 7 q \leqslant k \leqslant 7 q+3,
\end{array}\right. \\
b_{k}(M)=B_{k}(M), \quad k \neq m-1 \text { or } n-1, \\
b_{m-1}(M)=\pi \sqrt{5}=B_{m-2}(M)<B_{m-1}(M)=\pi \sqrt{8},
\end{gathered}
$$

and

$$
b_{n-1}(M)=\pi \sqrt{8}=B_{n-2}(M)<B_{m-1}(M)=\pi \sqrt{17} .
$$

2.3. EXAmple. Let $M=\mathbf{R} P^{n}, n \geqslant 2$, with metric induced by the projection $S^{n} \rightarrow \mathbf{R} P^{n}$. The loopspace $\Omega \mathbf{R} P^{n} \sim S^{0} \times \Omega S^{n}$ and the Morse theory is easy to calculate. We find that

$$
B_{k}^{\#}\left(\mathbf{R} P^{n}\right)=B_{k}\left(\mathbf{R} P^{n}\right)=q \pi, \quad(q-1)(n-1) \leqslant k<q(n-1) .
$$

Thus these numbers coincide with those of $S^{n}$. Since any two points of $\mathbf{R} P^{n}$ may be connected by a path of length no greater than $\pi / 2$,

$$
b_{0}\left(\mathbf{R} P^{n}\right)=\pi / 2<\pi=B_{0}\left(\mathbf{R} P^{n}\right) .
$$

The standard application of Theorems 1.10 and 1.15 yields that

$$
b_{k}\left(\mathbf{R} P^{n}\right)=b_{k}\left(S^{n}\right), \quad k \geqslant 1 \text {. }
$$

We note that all three of these examples are consistent with our conjecture that $b_{k}$ is always equal to either $B_{k}$ or $B_{k-1}$. The next examples illustrate the gaps that may occur between our upper bounds on $B_{k}^{\#}(M)$ and our lower bounds on $B_{k}(M)$.

2.4. ExAmple. Let $M$ be the ellipsoid in $\mathbf{R}^{3}$ given by the equation $(x / a)^{2}+y^{2}+$ $z^{2}=1$, with $a>1$. If $x=(0,1,0)$ and $x^{\prime}$ is any sufficiently nearby point, then for small values of $m$ the space $\Omega_{2 \pi m}\left(x, x^{\prime}\right)$ is not connected, in similar fashion to Example 1.8. But the Ricci curvature is everywhere positive on $M$. By well-known Morse theory, see e.g. [8], for every $k$ there exists $\lambda$ such that $i_{*}$ is an isomorphism on $\pi_{k}$ for all nonconjugate $x, x^{\prime} \in M$. In this case there is a gap between the value of $\lambda$ for which $i_{*}$ becomes onto and the value for which it becomes isomorphic. Although $B_{k}^{\#}(M)$ and $B_{k}(M)$ must lie in this range of values, our present methods do not tell us where.

2.5. EXAMPLE. Let $M=G$, a Lie group with a bi-invariant metric. For each pair $x, x^{\prime}$ of nonconjugate points in $G$, all geodesics from $x$ to $x^{\prime}$ have even index [1]. It follows that if $i_{*}: H_{k}\left(\Omega_{\lambda}\left(x, x^{\prime}\right)\right) \rightarrow H_{k}\left(\Omega\left(x, x^{\prime}\right)\right)$ is onto for some $k$ and $\lambda$, then $i_{*}$ is an isomorphism on $H_{k}$ for all $\lambda^{\prime} \geqslant \lambda$. We thus obtain

$$
B_{k}^{\#}(G)=B_{k}(G)=\inf \left\{\lambda: i_{*} \text { is onto for } \lambda \text { and all } x, x^{\prime} \in G\right\} .
$$

Since these numbers are completely determined by the Morse theory, we may turn to the $b_{k}(G)$. If $x$ and $x^{\prime}$ are nonconjugate points then $H_{k}\left(\Omega_{\lambda}\left(x, x^{\prime}\right)\right)=0=$ $H_{k}\left(\Omega\left(x, x^{\prime}\right)\right)$ for all odd $k$. It follows that $B_{2 n+1}(G)=B_{2 n}(G)$ for all $n$. Suppose 
that $\lambda$ is such that $B_{2 n}(G)>\lambda$. Then $i_{*}$ is not onto in degree $k=2 n$ for some $\left(x, x^{\prime}\right)$. Thus $b_{k}(G)>\lambda$ for $k \geqslant 2 n+1$, so $b_{2 n+1}(G) \geqslant B_{2 n}(G)$ and hence equals $B_{2 n+1}(G)$.

The number $b_{2 n}(G)$ needs more information. We recall that $G$ is the rational homotopy type of the product of odd-dimensional spheres, say $G \sim{ }_{0} S^{2 n_{1}-1} \times$ $\cdots \times S^{2 n_{i}-1}$. Thus if $2 n \notin\left\{2 n_{1}-2, \ldots, 2 n_{i}-2\right\}$, the image of the Hurewicz map on $\Omega G$ in dimension $2 n$ must be zero. It follows that in all but this finite set of cases $b_{2 n}(G)=B_{2 n}(G)$.

In the next two sections we prove the theorems of $\S \mathrm{I}$.

III. Upper bounds. We begin by fixing some notation. If $x, y \in M$, let $\operatorname{dist}(x, y)$ be the length of a minimal geodesic from $x$ to $y$. Let $\rho_{M}$ be the injectivity radius of $M$, so that if $\operatorname{dist}(x, y)<\rho_{m}$ there exists a unique minimal geodesic $m(x, y)$ from $x$ to $y$. Let $B(x, \varepsilon)$ denote the open $\varepsilon$-ball with respect to the metric disc.

In the proof of Theorem 1.14 we shall replace the space $M^{I}$ by its subspace $E$ consisting of piecewise smooth paths of constant speed. If $A_{1}, A_{2} \subset M, E\left(A_{1}, A_{2}\right)$ will be the subspace of $E$ consisting of paths whose endpoints lie in $A_{1}$ and $A_{2}$, respectively. The topology of $E$ is that of [1], so that path length becomes a continuous function on $E$. The projection $E \rightarrow M \times M$ is fiber-homotopy equivalent to $M^{I} \rightarrow M \times M$. If $\alpha, \beta \in E$ and $\alpha(1)=\beta(0)$, let $\alpha * \beta$ denote the concatenation of $\alpha$ with $\beta$ reparametrized so as to have constant speed. If $\alpha \in E$ and $0 \leqslant s \leqslant t \leqslant 1$, then $\alpha[s, t]$ is the portion of $\alpha$ from $\alpha(s)$ to $\alpha(t)$ reparametrized so as to be in $E$.

Theorem 1.14 is an immediate consequence of the following somewhat stronger theorem about $E_{\lambda}=E \cap\left(M^{I}\right)_{\lambda}$.

3.1. ThEOREM. Let $k \geqslant 1$ and $\lambda>0$. If there exists a dense set of points $\Sigma=$ $\left\{\left(x, x^{\prime}\right)\right\} \subset M \times M$ such that for every $\varepsilon^{\prime}<\rho_{m}$ there exists $\varepsilon<\varepsilon^{\prime}$ such that $\pi_{i}\left(E_{\lambda+\varepsilon}\left(x, x^{\prime}\right)\right) \rightarrow \pi_{i}\left(E\left(x, x^{\prime}\right)\right)$ is an isomorphism for all $i<k$, then $E_{\lambda} \rightarrow M \times M$ has the $\mathrm{XCHP}(k-1)$.

The proof will use the following two lemmas.

3.2. LeMmA. If $\lambda>0, \varepsilon<\frac{1}{2} \rho_{M}$ and $x, x^{\prime} \in M$, then the map

$$
\Phi: E_{\lambda}\left(B(x, \varepsilon), B\left(x^{\prime}, \varepsilon\right)\right) \rightarrow E_{\lambda+2 \varepsilon}\left(x, x^{\prime}\right)
$$

given by

$$
\Phi(\omega)=m(x, \omega(0)) * \omega * m\left(\omega(1), x^{\prime}\right)
$$

is a homotopy equivalence.

Proof. This is just a restatement of Lemma 1.6 of [3] generalized from the special case $M=S^{n}$. The formulas given there show that a homotopy inverse to $\Phi$ is given by $\Psi(\alpha)=\alpha[\delta, 1-\delta]$, where $\delta=\varepsilon /(\lambda+2 \varepsilon)$.

3.3. Lemma. Let $\varepsilon^{\prime}, \delta^{\prime}>0$ and $\left(x, x^{\prime}\right) \in \Sigma$. Under the hypotheses of Theorem 3.1, there exists $\varepsilon<\varepsilon^{\prime}$ such that if $\left(p, p^{\prime}\right) \in \Sigma \cap\left(B(x, \varepsilon) \times B\left(x^{\prime}, \varepsilon\right)\right)$ there exists $\delta<$ 
$\min \left\{\varepsilon-\operatorname{dist}(p, x), \varepsilon-\operatorname{dist}\left(x^{\prime}, p^{\prime}\right), \delta^{\prime}\right\}$ such that

$$
\pi_{i}\left(E_{\lambda}\left(B(p, \delta), B\left(p^{\prime}, \delta\right)\right)\right) \rightarrow \pi_{i}\left(E_{\lambda}\left(B(x, \varepsilon), B\left(x^{\prime}, \varepsilon\right)\right)\right)
$$

is an isomorphism for $0 \leqslant i<k$.

Proof. If $\varepsilon, \delta$ satisfy the above inequalities, we have the diagram

$$
\begin{array}{ccc}
E_{\lambda}\left(B(p, \delta), B\left(p^{\prime}, \delta\right)\right) & \stackrel{\Phi_{p}}{\rightarrow} & E_{\lambda+2 \delta}\left(p, p^{\prime}\right) \\
f \downarrow & & \downarrow g \\
E_{\lambda}\left(B(x, \varepsilon), B\left(x^{\prime}, \varepsilon\right)\right) & \stackrel{\Phi_{x}}{\rightarrow} & E_{\lambda+2 \varepsilon}\left(x, x^{\prime}\right)
\end{array}
$$

in which $f$ is inclusion and $g(\alpha)=m(x, p) * \alpha * m\left(p^{\prime}, x^{\prime}\right)$. This diagram is not commutative, but it is homotopy-commutative, since the map $\Gamma$ given by

$$
\begin{aligned}
\Gamma(\alpha, s)= & m(x, m(p, \alpha(0)(s))) * m(p, \alpha(0))[s, 1] * \alpha \\
& * m\left(\alpha(1), p^{\prime}\right)[0,1-s] * m\left(m\left(\alpha(1), p^{\prime}\right)(1-s), x^{\prime}\right)
\end{aligned}
$$

has

$$
\Gamma(\alpha, 0)=m(x, p) * m(p, \alpha(0)) * \alpha * m\left(\alpha(1), p^{\prime}\right) * m\left(p^{\prime}, x^{\prime}\right)=g\left(\Phi_{p}(\alpha)\right),
$$

and

$$
\Gamma(\alpha, 1)=m(x, \alpha(0)) * \alpha * m\left(\alpha(1), x^{\prime}\right)=\Phi_{x}(\alpha)=\Phi_{x}(f(\alpha)) .
$$

We now choose $\varepsilon, \delta$ such that

$$
\pi_{i}\left(E_{\lambda+2 \delta}\left(p, p^{\prime}\right)\right) \rightarrow \pi_{i}\left(E\left(p, p^{\prime}\right)\right)
$$

and

$$
\pi_{i}\left(E_{\lambda+2 \varepsilon}\left(x, x^{\prime}\right)\right) \rightarrow \pi_{i}\left(E\left(x, x^{\prime}\right)\right)
$$

are isomorphic for $0 \leqslant i<k$, as guaranteed by hypothesis.

We next extend $g$ to $g^{\prime}: E\left(p, p^{\prime}\right) \rightarrow E\left(x, x^{\prime}\right)$ by the same formula as $g$. This map is clearly a homotopy equivalence (its inverse is given by $\beta \rightarrow m(p, x)$ $\left.* \beta * m\left(x^{\prime}, p^{\prime}\right)\right)$.

Thus in the diagram

$$
\begin{array}{ccc}
E_{\lambda+2 \delta}\left(p, p^{\prime}\right) & \rightarrow & E\left(p, p^{\prime}\right) \\
\downarrow & & \downarrow \\
E_{\lambda+2 \varepsilon}\left(x, x^{\prime}\right) & \rightarrow & E\left(x, x^{\prime}\right)
\end{array}
$$

the top, bottom and right-hand arrows induce isomorphisms on $\pi_{i}, 0 \leqslant i<k-1$. Consequently $g$ and hence $f$ also induce isomorphisms.

Proof of Theorem 3.1. The proof follows standard lines. By the "bridge" argument of [9] it suffices to consider $X$ a polyhedron. We content ourselves with a description of the inductive step of an induction on the skeleta of $X$; cf. [5]. 
We suppose given a map

$$
H:\left(\Delta^{j} \times I, \Delta^{j} \times\{1\}\right) \rightarrow\left(B(x, \varepsilon) \times B\left(x^{\prime}, \varepsilon\right), U\right),
$$

where $\Delta^{j}$ is a closed $j$-simplex, $\varepsilon$ is as in Lemma 3.3, and $U$ is an open subset of $B(x, \varepsilon) \times B\left(x^{\prime}, \varepsilon\right)$. Let $b$ be the barycenter of $\Delta^{j}$ and $\left(p, p^{\prime}\right)=H(b, 1)$. We may adjust $\left(p, p^{\prime}\right)$ very slightly so that $\left(p, p^{\prime}\right) \in \Sigma$; hence there is $\delta$ as in Lemma 3.3 such that $B(p, \delta) \times B\left(p^{\prime}, \delta\right) \subset U$. Let $\sigma^{j}$ be an open subsimplex of $\Delta^{j}$ such that $b \in \Delta^{j}$ and

$$
H\left(\sigma^{j} \times\{1\}\right) \subset B(p, \delta) \times B\left(p^{\prime}, \delta\right) .
$$

We assume inductively that on the complex

$$
L=\left(\Delta^{j} \times\{0\}\right) \cup\left(\operatorname{Bd}\left(\Delta^{j}\right) \times I\right) \cup\left(\left(\Delta^{j} \backslash \sigma^{j}\right) \times\{1\}\right)
$$

we are given a map $\hat{H}: L \rightarrow E_{\lambda}$ such that

$$
\left(p \hat{H}(L), p \hat{H}\left(\operatorname{Bd}\left(\sigma^{j}\right) \times\{1\}\right)\right) \subset\left(B(x, \varepsilon) \times B\left(x^{\prime}, \varepsilon\right), B(p, \delta) \times B\left(p^{\prime}, \delta\right)\right) .
$$

Thus $\hat{H}$ defines an element of

$$
\pi_{j}\left(E_{\lambda}\left(B(x, \varepsilon), B\left(x^{\prime}, \varepsilon\right)\right), E_{\lambda}\left(B(p, \delta), B\left(p^{\prime}, \delta\right)\right)\right),
$$

which group is zero of $0 \leqslant j<k-1$.

Hence $\hat{H}$ extends to map $\left(\Delta^{j} \times I, \Delta^{j} \times\{1\}\right)$ into

$$
\left(E_{\lambda}\left(B(x, \varepsilon), B\left(x^{\prime}, \varepsilon\right)\right), E_{\lambda}\left(B(p, \delta), B\left(p^{\prime}, \delta\right)\right)\right)
$$

and the inductive step is completed.

The next lemma will be used in the proof of Theorem 1.15. We fix some more notation. If $x, x^{\prime} \in M$ and $\varepsilon<\rho_{M}$, let $U\left(x, x^{\prime} ; \varepsilon\right)=B(x, \varepsilon) \times B\left(x^{\prime}, \varepsilon\right)$.

3.4. Lemma. Let $x, x^{\prime} \in M, k \geqslant 0$ and $\nu>0$ be such that $\pi_{i}\left(E\left(x, x^{\prime}\right), E_{\nu}\left(x, x^{\prime}\right)\right)=$ 0 for $i \leqslant k$. Let $Y$ be a complex of dimension $k$ or less and $W \subset Y$ a subcomplex. Let $U=U\left(x, x^{\prime} ; \varepsilon\right)$. Suppose that $g:(Y, W) \rightarrow\left(E(U), E_{\nu-2 \varepsilon}(U)\right)$ is a map. Then there is a vertical homotopy

$$
H:(Y, W) \times I \rightarrow\left(E(U), E_{\nu+2 \varepsilon}(U)\right)
$$

such that $H(y, 0)=g(y)$ and $H(Y \times\{1\}) \subset E_{\nu+2 \varepsilon}(U)$.

Proof. Define $\hat{g}:(Y, W) \rightarrow\left(E\left(x, x^{\prime}\right), E_{\nu}\left(x, x^{\prime}\right)\right)$ by

$$
\hat{g}(y)=m(x, g(y)(0)) * g(y) * m\left(g(y)(1), x^{\prime}\right) .
$$

By hypothesis, there exists

$$
\hat{H}:(Y, W) \times I \rightarrow\left(E\left(x, x^{\prime}\right), E_{\nu}\left(x, x^{\prime}\right)\right)
$$

such that $\hat{H}(y, 0)=\hat{g}(y)$ and $\hat{H}(Y \times\{1\}) \subset E_{\nu}\left(x, x^{\prime}\right)$. We now define

$$
\bar{H}(y, t)=m(g(y)(0), x) * \hat{H}(y, t) * m\left(x^{\prime}, g(y)(1)\right) \text {. }
$$


Then

$$
\begin{aligned}
\bar{H}(y, 0)= & m(g(y)(0), x) * m(x, g(y)(0)) \\
& * \hat{H}(y, t) * m\left(g(y)(1), x^{\prime}\right) * m\left(x^{\prime}, g(y)(1)\right) .
\end{aligned}
$$

Hence $g(y)$ is homotopic to $\bar{H}(y, 0)$ by shrinking down the end segments while keeping the ends fixed at $g(y)(0)$ and $g(y)(1)$. The required $H$ is the concatenation of this homotopy with $\bar{H}$.

3.5. Construction. We next suppose that $X$ is a complex of dimension $k$ or less and that $g: X \rightarrow E$ is a map. Let $\varepsilon>0$ and $\left\{\left(x, x^{\prime}\right)\right\}$ be an $\varepsilon$-dense set of pairs in $M \times M$. We may partition (a subdivision of) $X$ into subcomplexes $\left\{X_{\alpha}\right\}_{\alpha \in A}$ such that $p\left(g\left(X_{\alpha}\right)\right)$ is contained in some $U\left(x, x^{\prime} ; \varepsilon\right)$ and such that $A=A_{1} \cup \cdots \cup A_{k+1}$ where $X_{\alpha} \cap X_{\beta}=\varnothing$ if $\alpha, \beta \in A_{j}, \alpha \neq \beta$.

Theorem 1.15 will be an application of the next

3.6. TheOREM. Suppose $\Sigma=\left\{\left(x, x^{\prime}\right)\right\}$ is an $\varepsilon$-dense set of pairs in $M \times M$, and $\lambda, \varepsilon, k$ are such that

$$
\pi_{i}\left(E\left(x, x^{\prime}\right), E_{\lambda^{\prime}}\left(x, x^{\prime}\right)\right)=0
$$

for $0 \leqslant i \leqslant k,\left(x, x^{\prime}\right) \in \Sigma$ and $\lambda<\lambda^{\prime} \leqslant \lambda+(4 k+2) \varepsilon$. Let $X$ be a complex of dimension $\leqslant k$ and $Z$ a subcomplex. If $g_{0}:(X, Z) \rightarrow\left(E, E_{\lambda}\right)$ is any map, then there is a vertical deformation

$$
H:(X, Z) \times I \rightarrow\left(E, E_{\lambda+4(k+1) \varepsilon}\right)
$$

such that $H(x, 0)=g_{0}(x)$, and $H(X \times\{1\}) \subset E_{\lambda+4(k+1) \varepsilon}$.

Proof. We construct a sequence of maps $g_{j}(j=0,1, \ldots, k+1)$ as follows. Let $X$ be partitioned as in 3.5 and define

$$
B_{j}=\bigcup\left\{X_{\alpha} \mid \alpha \in A_{j}\right\} .
$$

Let $C_{j}=\cup_{i \leqslant j} B_{i}$. We suppose that $g_{i}$ is defined for $0 \leqslant i<j$ such that $g_{0}$ is the hypothesized $g_{0}$ and if $1 \leqslant i<j$ that

$$
g_{i}:\left(X, C_{i} \cup Z\right) \rightarrow\left(E, E_{\lambda+4 i \varepsilon}\right)
$$

is vertically homotopic to $g_{0}$ as a map of pairs.

For every $\alpha \in A_{i}$, let $\hat{X}_{\alpha}$ be a "halo complex" of $X_{\alpha}$ in $X$ such that if $\alpha \neq \beta$ then $\hat{X}_{\alpha} \cap \hat{X}_{\beta}=\varnothing$ and such that $p\left(g_{0}\left(\hat{X}_{\alpha}\right)\right)$ is contained in some $U\left(x, x^{\prime} ; \varepsilon\right)$ which we name $U_{\alpha}$. Thus we have $\eta_{\alpha}: X \rightarrow I$ such that $\eta_{\alpha}\left(X_{\alpha}\right)=1$ and $\eta_{\alpha}\left(X \backslash \hat{X}_{\alpha}\right)=0$.

We now apply Lemma 3.4, taking $U=U_{\alpha}, Y=\hat{X}_{\alpha}, W=\hat{X}_{\alpha} \cap\left(C_{j-1} \cup Z\right)$, $g=g_{j-1} \mid \hat{X}_{\alpha}$ and $\nu=\lambda+(4 j-2) \varepsilon$.

Let $H_{\alpha}:\left(\hat{X}_{\alpha}, \hat{X}_{\alpha} \cap\left(C_{j-1} \cup Z\right)\right) \times I \rightarrow\left(E\left(U_{\alpha}\right), E_{\lambda+4 j \varepsilon}\left(U_{\alpha}\right)\right)$ be the homotopy given by 3.4 .

Now define

$$
g_{j}(x)=\left(\begin{array}{ll}
H_{\alpha}\left(x, \eta_{\alpha}(x)\right) & \text { if } x \in \hat{X}_{\alpha}, \alpha \in A_{j}, \\
g_{j-1}(x) & \text { otherwise. }
\end{array}\right.
$$

Clearly $g_{j}$ satisfies the inductive hypothesis. Completing the induction, we arrive at 


$$
g_{k+1}:(X, Z) \rightarrow\left(E, E_{\lambda+4(k+1) \varepsilon}\right)
$$

which is vertically homotopic to $g_{0}$ as a map of pairs.

Proof of Theorem 1.15. Lifting a map $(f, g): X \rightarrow M \times M$ to $E_{\lambda}$ produces a homotopy from $f$ to $g$ of width less than $\lambda$. Suppose that $X$ is a $k$-dimensional complex, that $A \subset X$, and that $H: X \times I \rightarrow M$ is a homotopy such that $|H|_{A} \mid<\lambda$. By Theorem 1.14 we may deform $H \mid\left(X^{(k-1)} \times I\right)$ (rel. $\left.X^{(k-1)} \times\{0,1\}\right)$ to have width less than $\lambda$ in such a way that $H \mid\left(A^{(k-1)} \times I\right)$ is deformed through homotopies of with $<\lambda$. The proof of the homotopy extension theorem together with a patching argument similar to that of Theorem 3.7 of [10] shows that we may extend this deformation to $A \times I$ to obtain a deformation of

$$
H \mid\left(X^{(k-1)} \cup A\right) \times I \quad\left(\text { rel. }\left(X^{(k-1)} \cup A\right) \times\{0,1\}\right)
$$

such that $H \mid(A \times I)$ is deformed through homotopies of width $<\lambda$. Another application of the homotopy extension theorem allows us to extend this deformation (relative to $X \times\{0,1\})$ to all of $X \times I$. Thus we may assume without loss of generality that $H$ satisfies this condition. Thus $H$ may be regarded as a map of pairs $H:\left(X, X^{(k-1)} \cup A\right) \rightarrow\left(E, E_{\lambda}\right)$. For $\delta>0$, choose an $\varepsilon$-dense set $\left\{\left(x, x^{\prime}\right)\right\} \subset M \times M$ for some $\varepsilon<\delta /(4 k+4)$. We apply Theorem 3.6 to these data to obtain

$$
H^{\prime}: X \rightarrow E_{\lambda+4(k+1) \varepsilon} \subset E_{\lambda+\delta}
$$

that is vertically homotopic to $H$. Since this can be done for arbitrarily small $\delta$, $B_{k}(M) \leqslant \lambda$.

Proof of Theorem 1.17. Let $0<\varepsilon<\rho_{M}$. Let $K$ be a $k$-dimensional complex and let $H: K \times I \rightarrow M$ be a homotopy between maps $f, g: K \rightarrow M$. We subdivide $K$ so that the image of each simplex under the map $(f, g): K \rightarrow M \times M$ is contained in $U\left(x, x^{\prime} ; \varepsilon / 8\right)$ for some $\left(x, x^{\prime}\right) \in \Sigma$.

Since $B_{k-1}(M) \leqslant \lambda$ we may deform $H \mid\left(K^{(k-1)} \times I\right)$, keeping endpoints fixed, to a homotopy $\hat{H}$ of width $\leqslant \lambda+\varepsilon / 4$. We use the homotopy extension property to extend $\hat{H}$ to all of $K \times I$ as a homotopy from $f$ to $g$.

Let $\Delta$ be a $k$-simplex of $K$ and suppose that $(F, g)(\Delta) \subset U=U\left(x, x^{\prime} ; \varepsilon / 8\right)$. Now $\hat{H} \mid(\Delta \times I)$ determines a map $\hat{h}:(\Delta, \operatorname{Bd}(\Delta)) \rightarrow\left(E(U), E_{\lambda+\varepsilon / 4}(U)\right)$.

Since $\left(E(U), E_{\lambda+\varepsilon / 4}(U)\right) \sim\left(E\left(x, x^{\prime}\right), E_{\lambda+\varepsilon / 2}\left(x, x^{\prime}\right)\right)$, the hypothesis guarantees that $\hat{h} \mid \operatorname{Bd}(\Delta)$ extends to a map $\tilde{h}: \Delta \rightarrow E_{\lambda+\varepsilon / 4}(U)$. If $\tilde{H}: \Delta \times I \rightarrow U$ is the homotopy determined by $\tilde{h}$, then $\tilde{H}$ is no longer a homotopy from $f \mid \Delta$ to $g \mid \Delta$; but since $f(\Delta)$ and $\tilde{H}(\Delta \times 0)$ (resp. $g(\Delta)$ and $\tilde{H}(\Delta \times 1)$ ) are both in $B(x, \varepsilon / 8)$ (resp. $B\left(x^{\prime}, \varepsilon / 8\right)$ ), we may compose with geodesics of length $<\varepsilon / 4$ at each end of $\tilde{H}$ to obtain a homotopy $\bar{H}$ of width $<\lambda+3 \varepsilon / 4$ from $f \mid \Delta$ to $g \mid \Delta$. Since $\bar{H}$ agrees with $\hat{H}$ on $\operatorname{Bd}(\Delta) \times I$, we may carry out this process on each $k$-simplex of $K$ to extend $\hat{H} \mid\left(K^{(k-1)} \times I\right)$ to $\bar{H}: K \times I \rightarrow M$, a homotopy from $f$ to $g$ of width $<\lambda+3 \varepsilon / 4$. Hence $b_{k}(M) \leqslant \lambda$.

IV. Lower bounds. The lower bounds we shall obtain require only null-homotopic maps and homotopies to the constant map. Hence we shall concentrate in this section on the subspaces $P M(x)$ of $M^{I}$ consisting of those paths emanating from 
$x \in M$. We shall use the principal action $\eta: \Omega(x, x) \times P M(x) \rightarrow P M(x)$ given by $\eta(\alpha, \omega)=\alpha * \omega$.

Proofs of TheOrems 1.10 AND 1.11. For the first part of Theorem 1.10, suppose that $x, x^{\prime} \in M, \lambda>0$, and $k$ are such that $i_{*}: H_{k}\left(\Omega_{\lambda}\left(x, x^{\prime}\right)\right) \rightarrow H_{k}\left(\Omega\left(x, x^{\prime}\right)\right)$ fails to be onto. We may choose a $k$-dimensional complex $K$ and map $f: K \rightarrow \Omega\left(x, x^{\prime}\right)$ such that $f_{\#}$ is epimorphic on $H_{k}$. If we regard $f$ as a homotopy between the constant maps at $x$ and $x^{\prime}$, respectively, then a deformation of the homotopy keeping endpoints fixed amounts simply to a homotopy of $f$ in $\Omega\left(x, x^{\prime}\right)$. If $B_{k}(M)<\lambda$, we could deform $f$ into $\Omega_{\lambda}\left(x, x^{\prime}\right)$, contradicting the fact that $f_{\#}$ is onto. Hence $B_{k}(M) \geqslant \lambda$. (Clearly this portion of the proof applies also to $\pi_{k}$, and indeed to any homotopy functor for which the inclusion of the $k$-skeleton into a complex induces an epimorphism.) Since this bound uses only constant maps, the calculation is identical to that of Gromov [7].

To show that $b_{k+1}(M) \geqslant \lambda$, we begin with the map $f: K \rightarrow \Omega\left(x, x^{\prime}\right)$ of the preceding paragraph, then extend in any fashion to the cone on $K$ to obtain $\bar{f}$ : $C K \rightarrow P M(x)$. We regard $\bar{f}$ as a homotopy from the constant map $[x]$ at $x$ to the map $p \bar{f}: C K \rightarrow M$. Since $\bar{f} \mid K=f$, which does not factor through $\Omega_{\lambda}\left(x, x^{\prime}\right)$, the width of $\bar{f}$ is certainly greater than or equal to $\lambda$. We shall show that if $\bar{g}$ is any homotopy from $[x]$ to $p \bar{f}$ regarded as a map $\bar{g}$ : $C K \rightarrow P M(x)$ then $g=\bar{g} \mid K$ does not factor through $\Omega_{\lambda}\left(x, x^{\prime}\right)$.

Now any lift of $p \bar{f}$ to $P M(x)$ is vertically homotopic to one of the form

$$
C K \stackrel{\Delta}{\rightarrow} C K \times C K \stackrel{\alpha \times \bar{f}}{\rightarrow} \Omega(x, x) \times P M(x) \stackrel{\eta}{\rightarrow} P M(x) .
$$

Thus $g_{\#}: H_{k}(K) \rightarrow H_{k}\left(\Omega\left(x, x^{\prime}\right)\right)$ factors as

$$
\begin{aligned}
& H_{k}(K) \quad \stackrel{g_{\#}}{\rightarrow} \quad H_{k}\left(\Omega\left(x, x^{\prime}\right)\right) \\
& \downarrow \Delta_{\#} \\
& H_{k}(K \times K) \rightarrow H_{k}(C K \times K) \stackrel{(\alpha \times f)_{\#}}{\rightarrow} \quad H_{k}\left(\Omega(x, x) \times \Omega\left(x, x^{\prime}\right)\right) \\
& H_{0}(C K) \otimes H_{k}(K) \stackrel{\alpha \otimes f_{\#}}{\rightarrow} \quad H_{0}(\Omega(x, x)) \otimes H_{k}\left(\Omega\left(x, x^{\prime}\right)\right)
\end{aligned}
$$

where $\bar{\eta}=\eta \mid\left(\Omega(x, x) \times \Omega\left(x, x^{\prime}\right)\right)$.

Thus $g_{\#}$ differs from $f_{\#}$ only by Pontryagin multiplication by the element $\alpha_{\#}(\iota)$, where $\iota$ generates $H_{0}(C K)$. In particular $g_{\#}$ is onto, so $\bar{g}$ cannot factor through $P_{\lambda} M(x)$. Since $\operatorname{dim}(C K)=k+1, b_{k+1}(M) \geqslant \lambda$.

To consider $b_{k}(M)$, we consider the $k$-dimensional complex $\hat{K}=K \cup C\left(K^{(k-1)}\right)$ and $\hat{f}=\bar{f} \mid \hat{K}$. Again, any lifting $\hat{g}: \hat{K} \rightarrow P M(x)$ of $p \hat{f}$ differs from $\hat{f}$, up to vertical homotopy, by the principal action of a map $\alpha: \hat{K} \rightarrow \Omega(x, x)$. Hence if $g=\hat{g} \mid K$, we 
factor $g_{\#}$ (using the fact that $\hat{K}$ is homotopic to a bouquet of spheres) as

$$
\begin{array}{ccc}
H_{k}(K) \stackrel{\Delta_{\#}}{\rightarrow} H_{k}(\hat{K} \times K) & \stackrel{\sim}{\rightarrow} & H_{k}(\hat{K}) \otimes H_{0}(K) \otimes H_{0}(\hat{K}) \otimes H_{k}(K) \\
\downarrow(\alpha \times f)_{\#} & & \downarrow \alpha_{\#} \otimes f_{\#} \\
H_{k}\left(\Omega(x, x) \times \Omega\left(x, x^{\prime}\right)\right) & \leftarrow & H_{k}(\Omega(x, x)) \otimes H_{k}\left(\Omega\left(x, x^{\prime}\right)\right) \oplus H_{0}\left(\Omega\left(x, x^{\prime}\right)\right) \otimes H_{k}\left(\Omega\left(x, x^{\prime}\right)\right) \\
\bar{\eta}_{\#} \downarrow & & \\
H_{k}\left(\Omega\left(x, x^{\prime}\right)\right. & &
\end{array}
$$

The right-hand groups are as in the preceding case, hence the only difficulty in $g_{\#}$ being onto must arise through

$$
H_{k}(\hat{K}) \otimes H_{0}(K) \stackrel{\alpha_{\#} \otimes f_{\#}}{\rightarrow} H_{k}(\Omega(x, x)) \otimes H_{0}\left(\Omega\left(x, x^{\prime}\right)\right) .
$$

Since $\alpha_{\#}\left(H_{k}(\hat{K})\right)$ consists of spherical classes, the composition $\rho g_{\#}$ is onto the group $H_{k}\left(\Omega\left(x, x^{\prime}\right)\right) /$ spherical classes $\}$. Thus $\hat{g}$ cannot have its image in $P_{\lambda} M(x)$ and so $b_{k}(M) \geqslant \lambda$.

Proofs of TheOrems 1.10(c) AND 1.11(b). We follow the general outline of the previous proof except that we choose $K$ to be a $(k+1)$-dimensional complex such that $f: K \rightarrow \Omega\left(x, x^{\prime}\right)$ induces an isomorphism on $H_{k}$. Setting $\hat{K}=K \cup C\left(K^{(k)}\right)$, we again extend $f$ to $\hat{f}:(\hat{K}, K) \rightarrow\left(P M(x), \Omega\left(x, x^{\prime}\right)\right)$. If $\hat{g}: \hat{K} \rightarrow P M(x)$ is any other lift of $p \hat{f}$ and $g=\hat{g} \mid K$, then $g_{\#}$ factors as in the previous diagram. Since in the present case, $\hat{K}$ is homotopic to a wedge of $(k+1)$-spheres, $g_{\#}=f_{\#}$ on $H_{k}$. If $g(K) \subset$ $\Omega_{\lambda}\left(x, x^{\prime}\right)$, then we would have the commutative triangle:

$$
\begin{array}{ccc}
H_{k}(K) \stackrel{g_{\#}}{\rightarrow} & H_{k}\left(\Omega\left(x, x^{\prime}\right)\right) \\
\stackrel{y}{H_{k}\left(\Omega_{\lambda}\left(x, x^{\prime}\right)\right)} & & \nearrow i_{\#}
\end{array}
$$

Hence $i_{\#}$ admits a section.

A similar diagram for $\pi_{k}$ yields 1.11(b).

Proof OF COROLlary 1.19. The hypotheses of Theorem 1.15 guarantee that both $\pi_{j}\left(\Omega\left(x, x^{\prime}\right), \Omega_{\lambda^{\prime}}\left(x, x^{\prime}\right)\right)$ and $H_{j}\left(\Omega\left(x, x^{\prime}\right), \Omega_{\lambda^{\prime}}\left(x, x^{\prime}\right)\right)$ are trivial for $\lambda^{\prime}>\lambda$ and $j \leqslant k$. Thus the relative Hurewicz morphism

$$
h: \pi_{k+1}\left(\Omega\left(x, x^{\prime}\right), \Omega_{\lambda^{\prime}}\left(x, x^{\prime}\right)\right) \rightarrow H_{k+1}\left(\Omega\left(x, x^{\prime}\right), \Omega_{\lambda^{\prime}}\left(x, x^{\prime}\right)\right)
$$

is an isomorphism. Consequently there arises the following (Mayer-Vietoris) exact sequence:

$$
\begin{aligned}
& \pi_{k+1}\left(\Omega\left(x, x^{\prime}\right)\right) \oplus H_{k+1}\left(\Omega_{\lambda^{\prime}}\left(x, x^{\prime}\right)\right) \stackrel{\alpha}{\rightarrow} H_{k+1}\left(\Omega\left(x, x^{\prime}\right)\right) \\
& \rightarrow \pi_{k}\left(\Omega_{\lambda^{\prime}}\left(x, x^{\prime}\right)\right) \stackrel{\beta}{\rightarrow} \pi_{k}\left(\Omega\left(x, x^{\prime}\right)\right) \oplus H_{k}\left(\Omega_{\lambda^{\prime}}\left(x, x^{\prime}\right)\right) .
\end{aligned}
$$


The kernel of $\beta$ is $\operatorname{ker}\left(i_{*}\right) \cap \operatorname{ker}(h)$. By exactness, the kernel of $\beta$ is also equal to the cokernel of $\alpha$. Thus $\operatorname{ker}\left(i_{*}\right) \cap \operatorname{ker}(h) \neq 0 \Rightarrow \alpha$ is not epic. By Theorem $1.10(\mathrm{~b})$, $b_{k+1}(M) \geqslant \lambda^{\prime}>\lambda$.

\section{REFERENCES}

1. R. Bott, Lectures on Morse theory, Harvard Univ. Press, Cambridge, Mass., 1960.

2. A. Calder and J. Siegel, On the width of homotopies, Topology 19 (1980), 209-220.

3. A. Calder, J. Siegel and F. Williams, The width of homotopies into spheres, Topology 21 (1982), 281-290.

4. D. Coram and P. Duvall, Approximate fibrations and a movability condition for maps, Pacific J. Math. 72 (1977), 41-56.

5. A. Dold and R. Thom, Quasifaserungen und unendliche symmetrische produckte, Ann. of Math. (2) 67 (1958), 239-281.

6. A. Dold, Partitions of unity in the theory of fibrations, Ann. of Math. (2) 78 (1963), 223-255.

7. M. Gromov, Homotopical effects of dilatation, J. Differential Geom. 13 (1978), 303-310.

8. J. Milnor, Morse theory, Ann. of Math. Studies, No. 51, Princeton Univ. Press, Princeton, N. J., 1963.

9. K. Morita, Čech cohomology and covering dimension for toplogical spaces, Fund. Math. 87 (1975), $31-52$.

10. J. Siegel and F. Williams, Numerical invariants of homotopies into spheres, Pacific J. Math. 110 (1984), 417-428.

11. N. Steenrod, Fiber bundles, Princeton Univ. Press, Princeton, N. J., 1951.

Department of Mathematical Science, University of Missouri, St. Louis, Missouri 63121

Department of Mathematical Science, New Mexico State University, las Cruces, New MeXICO 88003 